\title{
THE EIGENVALUES AND EIGENSPACES OF SOME DISCRETE DIV- AND CURL-RELATED OPERATORS*
}

\author{
ERIC T. CHUNG $^{\dagger}$ AND JUN ZOU
}

\begin{abstract}
The eigenvalues and eigenspaces of some discrete div- and curl-related operators are investigated. The discrete operators give some good discrete analogues of the continuous counterparts and play an important role in developing finite volume schemes for solving div-curl equations and electromagnetic systems. Knowledge of the eigenvalues and eigenspaces is very useful in the numerical analysis of finite volume methods for electromagetic systems in nonhomogeneous media.
\end{abstract}

Key words. eigenvalues, eigenspaces, discrete div-operator, discrete curl-operator

AMS subject classifications. $65 \mathrm{~F} 15,78-08,65 \mathrm{~N} 25$

PII. S0895479801382483

1. Introduction. The aim of this paper is to find the explicit formulae for the complete eigenvalues and eigenspaces of some discrete div- and curl-related operators. These operators play a very important role in the finite volume approximation of the div-curl equations [7], [9] as well as of Maxwell's equations [3], [8]. Knowledge of the eigenvalues and eigenspaces is very useful in the numerical analysis of a newly developed finite volume method for electromagetic systems in nonhomogeneous media [3], [6].

We will mainly investigate three discrete operators: the discrete divergence, curlcurl, and Laplacian operators. We will see that these three discrete operators satisfy a relation that resembles the continuous counterpart. The main difficulty for the spectral analysis lies in the fact that all three components of a vector-valued function in $\mathbb{R}^{3}$ contribute to each component of the curl-curl operator, while this is not the case for the discrete Laplacian operator. Hence, the standard treatment for finding the eigenvalues and eigenspaces of a Laplacian operator does not work for the curlcurl operator. We will present a new approach for finding the complete eigenvalues and eigenspaces of the discrete curl-curl operator. As we will see, the spectra of the discrete curl-curl operator and the discrete Laplacian operator are similar, but their eigenspaces are different.

The paper is organized as follows. In section 2, we give the definitions of the discrete curl, divergence, and Laplacian operators. In section 3, we show an interesting relation among the three operators and study the complete eigenvalues and eigenspaces of the discrete operators. In section 4 , we present some applications of the discrete operators and their eigenvalues and eigenspaces.

2. Discrete differential operators. We consider a nonuniform triangulation, called the primal mesh, of the unit cube $\Omega=[0,1]^{3}$ by a set of small rectangular

* Received by the editors October 16, 2001; accepted for publication (in revised form) by M. Hanke October 14, 2002; published electronically March 13, 2003.

http://www.siam.org/journals/simax/24-4/38248.html

${ }^{\dagger}$ Department of Mathematics, University of California at Los Angeles, Los Angeles, CA 900951555 (tschung@math.ucla.edu).

${ }^{\ddagger}$ Department of Mathematics, The Chinese University of Hong Kong, Shatin, Hong Kong (zou@math.cuhk.edu.hk). The work of this author was supported by Hong Kong RGC grants CUHK4292/00P and CUHK4048/02P. 
subdomains, called primal elements. ${ }^{1}$ We denote by $N_{i}$ the number of primal elements in the $i$ th axis direction $(i=1,2,3)$. The faces, edges, and nodes of each primal element are called the primal faces, edges, and nodes, respectively. Then, we construct the dual mesh by connecting all the centers of primal elements; this gives another nonuniform triangulation of the domain $\Omega$. Each rectangular subdomain in the dual mesh is called a dual element. Dual faces, edges, and nodes are named as in the primal mesh. Later on, by an interior primal edge (face) we mean a primal edge (face) not completely lying on the boundary of $\Omega$. Moreover, we denote by $\sigma_{i}$ the $i$ th primal edge and by $\sigma_{j}^{\prime}$ the $j$ th dual edge. Here, we always use a primed form of a primal quantity to represent a dual quantity. For example, by $\kappa_{i}, \kappa_{j}^{\prime}, \tau_{r}$, and $\tau_{s}^{\prime}$ we mean the $i$ th primal face, $j$ th dual face, $r$ th primal element, and $s$ th dual element, respectively.

The above primal and dual meshes have an important internal relation: each interior primal face (edge) is perpendicular to and in one-to-one correspondence with a dual edge (face), and each interior primal node (element) is in one-to-one correspondence with a dual element (node). Now we assign each edge (both primal and dual) a direction in the way that each edge points to the positive axis direction and assign each primal (dual) face a direction such that it has the same direction as the corresponding dual (primal) edge.

Let $E, F$, and $T$ be the numbers of interior primal edges, faces, and nodes, respectively. Then by the aforementioned internal relation, we know $E, F$, and $T$ are also the numbers of dual faces, edges, and elements, respectively, and

$$
\begin{gathered}
E=\sum_{i=1}^{3} N_{i}\left(N_{i+1}-1\right)\left(N_{i+2}-1\right), \quad F=\sum_{i=1}^{3}\left(N_{i}-1\right) N_{i+1} N_{i+2}, \\
T=\left(N_{1}-1\right)\left(N_{2}-1\right)\left(N_{3}-1\right) .
\end{gathered}
$$

Here and in the subsequent sections we will use the convention that $N_{i}=N_{i-3}$ for $i>3$.

For a primal edge $\sigma_{j} \in \partial \kappa_{i}$, we say it is oriented positively along $\partial \kappa_{i}$ if its direction agrees with the direction of $\partial \kappa_{i}$ formed by the right-hand rule with the thumb pointing in the direction of $\kappa_{i}$. Otherwise, we say $\sigma_{j}$ is oriented negatively along $\partial \kappa_{i}$. In light of the Stokes theorem,

$$
\int_{\kappa_{i}}(\nabla \times \mathbf{u}) \cdot \mathbf{n} d \sigma=\int_{\partial \kappa_{i}} \mathbf{u} \cdot \mathbf{t} d l
$$

where $\mathbf{u}$ is a vector-valued function in $\mathbb{R}^{3}$, we define a discrete curl matrix $G$ by

$$
(G)_{i j}:=\left\{\begin{aligned}
1 & \text { if } \sigma_{j} \text { is oriented positively along } \partial \kappa_{i} \\
-1 & \text { if } \sigma_{j} \text { is oriented negatively along } \partial \kappa_{i} \\
0 & \text { if } \sigma_{j} \text { does not meet } \partial \kappa_{i}
\end{aligned}\right.
$$

Clearly $G$ is an $F \times E$ matrix, and $\operatorname{rank}(G)=E-T$ (cf. [9]). One of the goals of this paper is to find all the eigenvalues and eigenvectors of the $E \times E$ matrix $G^{T} G$, which is of rank $E-T . G^{T} G$ is symmetric positive semidefinite, so all its eigenvalues are nonnegative. Since the null space of $G^{T} G$ has dimension $T$, zero is an eigenvalue of $G^{T} G$ with multiplicity $T$. In other words, we need only to find all the remaining $E-T$ positive eigenvalues of $G^{T} G$.

\footnotetext{
${ }^{1}$ The results and techniques of this paper are directly applicable to treating the more general case, for instance, where the domain $\Omega$ is a union of some rectangular domains.
} 
For a dual face $\kappa_{j}^{\prime} \in \partial \tau_{i}^{\prime}$, we say it is oriented positively along $\partial \tau_{i}^{\prime}$ if its direction is pointing toward the outside of $\tau_{i}^{\prime}$. Otherwise, we say $\kappa_{j}^{\prime}$ is oriented negatively along $\partial \tau_{i}^{\prime}$. Initiated by the divergence theorem,

$$
\int_{\tau_{i}^{\prime}} \nabla \cdot \mathbf{u} d x=\int_{\partial \tau_{i}^{\prime}} \mathbf{u} \cdot \mathbf{n} d \sigma
$$

where $\mathbf{u}$ is a vector-valued function in $\mathbb{R}^{3}$, we define a discrete divergence matrix $B$ by

$$
(B)_{i j}:=\left\{\begin{aligned}
1 & \text { if } \kappa_{j}^{\prime} \text { is oriented positively along } \partial \tau_{i}^{\prime}, \\
-1 & \text { if } \kappa_{j}^{\prime} \text { is oriented negatively along } \partial \tau_{i}^{\prime}, \\
0 & \text { if } \kappa_{j}^{\prime} \text { does not meet } \partial \tau_{i}^{\prime} .
\end{aligned}\right.
$$

Then $B$ is a $T \times E$ matrix. It is known [9] that the $\operatorname{rank}$ of $B$ is $T$, and that $B G^{T}=0$, which is a discrete analogue of $\nabla \cdot(\nabla \times \mathbf{u})=0$.

Consider an interior primal edge $\sigma_{i}$. We say $\sigma_{k}$ is adjacent to $\sigma_{i}$ if both $\sigma_{i}$ and $\sigma_{k}$ lie on the same primal face or if their intersection is a single point. Clearly, for any interior primal edge having no intersection with $\partial \Omega$, it must have 6 adjacent primal edges. But for any interior primal edge having a nonempty intersection with $\partial \Omega$, the edge has only 5 adjacent primal edges. With these definitions in mind, we define an $E \times E$ discrete Laplacian matrix $A$ in the following way:

1. If $\sigma_{i}$ is an interior primal edge having no intersection with $\partial \Omega$, then

$$
(A)_{i j}:=\left\{\begin{aligned}
6 & \text { if } j=i, \\
-1 & \text { if } \sigma_{j} \text { is adjacent to } \sigma_{i}, \\
0 & \text { otherwise. }
\end{aligned}\right.
$$

2. If $\sigma_{i}$ is an interior primal edge having a nonempty intersection with $\partial \Omega$, then

$$
(A)_{i j}:=\left\{\begin{aligned}
5 & \text { if } j=i, \\
-1 & \text { if } \sigma_{j} \text { is adjacent to } \sigma_{i}, \\
0 & \text { otherwise. }
\end{aligned}\right.
$$

Note that this discrete Laplacian is different from the standard discrete Laplacian resulting from the discretization of the Laplacian operator by the second order central difference scheme. Instead, $B B^{T}$ is closer to the standard discrete Laplacian; see Theorem 3.4.

3. Eigenvalues and eigenspaces. This section will be devoted to our main results. For any $\mathbf{f} \in \mathbb{R}^{E}$, we will interpret its $i$ th component $f_{i}$ as its value on the $i$ th interior primal edge $\sigma_{i}$, as well as its value on the $i$ th dual face $\kappa_{i}^{\prime}$. We will often write $\mathbf{f}=\left(\mathbf{u}^{T}, \mathbf{v}^{T}, \mathbf{w}^{T}\right)^{T}$, where $\mathbf{u}$ ( $\mathbf{v}$ and $\mathbf{w}$, respectively) is a vector in $\mathbb{R}^{\frac{E}{3}}$ and each component of $\mathbf{u}$ corresponds to an interior primal edge parallel to the $x$-axis ( $y$-axis and $z$-axis, respectively).

Now, we are ready to present our first result, which is a discrete version of the well-known relation

$$
\nabla \times \nabla \times \mathbf{u}=\nabla(\nabla \cdot \mathbf{u})-\nabla^{2} \mathbf{u} .
$$

TheOrem 3.1. For the discrete curl, divergence, and Laplacian operators $G, B$, and $A$,

$$
G^{T} G=-B^{T} B+A .
$$




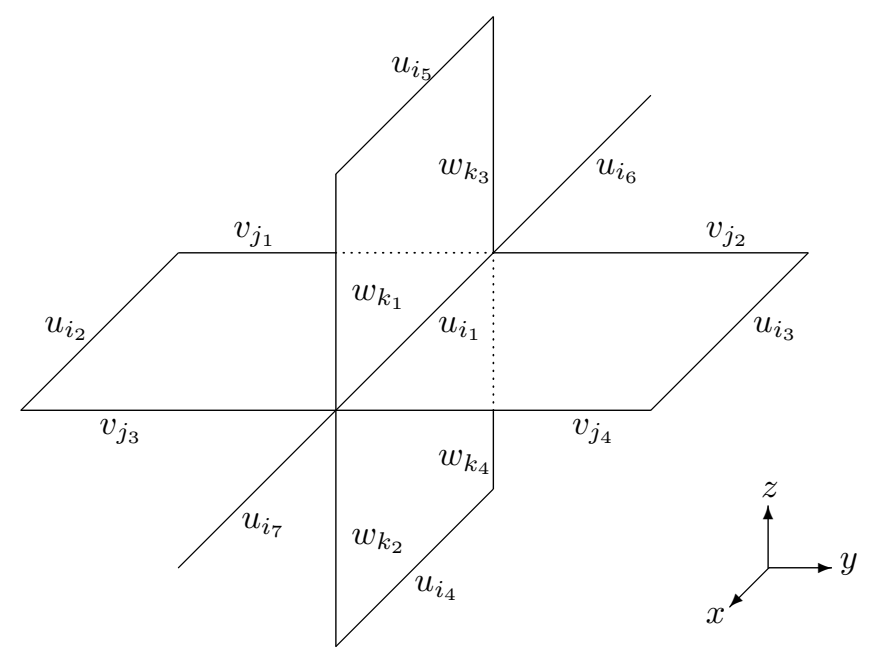

FIG. 3.1. An interior primal edge having no intersection with $\partial \Omega$ and its adjacent edges.

Proof. It suffices to show that for any vector $\mathbf{f}=\left(\mathbf{u}^{T}, \mathbf{v}^{T}, \mathbf{w}^{T}\right)^{T}$, we have

$$
G^{T} G \mathbf{f}=-B^{T} B \mathbf{f}+A \mathbf{f} .
$$

First we consider an interior primal edge $\sigma_{i}$ having no intersection with $\partial \Omega$; see Figure 3.1 .

Here, $u_{i_{1}}$ denotes a component of $\mathbf{u}$ corresponding to $\sigma_{i}$, which, without loss of generality, is assumed to be parallel to the $x$-axis. $u_{i_{s}}, s=2,3, \ldots, 7$, denote components of $\mathbf{u}$ corresponding to all adjacent edges of $\sigma_{i}$. Similarly, $v_{j_{r}}$ and $w_{k_{r}}$, $r=1,2,3,4$, are components of $\mathbf{v}$ and $\mathbf{w}$, respectively, corresponding to the primal edges parallel to the $y$-axis and $z$-axis. By the definitions of $G, B$, and $A$ and direct computations, we know the $i$ th components of $A \mathbf{f}, B^{T} B \mathbf{f}$, and $G^{T} G \mathbf{f}$ corresponding to $\sigma_{i}$ are, respectively, given by

$$
\begin{aligned}
(A \mathbf{f})_{i} & =6 u_{i_{1}}-u_{i_{2}}-u_{i_{3}}-u_{i_{4}}-u_{i_{5}}-u_{i_{6}}-u_{i_{7}} \\
\left(B^{T} B \mathbf{f}\right)_{i} & =\left(u_{i_{1}}-u_{i_{6}}+v_{j_{2}}-v_{j_{1}}+w_{k_{3}}-w_{k_{4}}\right)-\left(u_{i_{7}}-u_{i_{1}}+v_{j_{4}}-v_{j_{3}}+w_{k_{1}}-w_{k_{2}}\right), \\
\left(G^{T} G \mathbf{f}\right)_{i}=\left(4 u_{i_{1}}-u_{i_{2}}-u_{i_{3}}-u_{i_{4}}-u_{i_{5}}\right) & +\left(v_{j_{1}}-v_{j_{2}}-v_{j_{3}}+v_{j_{4}}\right) \\
& +\left(w_{k_{1}}-w_{k_{2}}-w_{k_{3}}+w_{k_{4}}\right) .
\end{aligned}
$$

This implies

$$
\left(G^{T} G \mathbf{f}\right)_{i}=-\left(B^{T} B \mathbf{f}\right)_{i}+(A \mathbf{f})_{i} .
$$

Now we consider an interior primal edge $\sigma_{i}$ having a single-point intersection with $\partial \Omega$. See Figure 3.2 below, where $P$ is the single-point intersection of $\sigma_{i}$ with $\partial \Omega$.

If one of the primal edges corresponding to the component $u_{i_{s}}, s=2,3,4,5$, lies on $\partial \Omega$, then we take $u_{i_{s}}$ to be zero since $\mathbf{f}$ does not contain any boundary component by definition. Then, the $i$ th components of $A \mathbf{f}, B^{T} B \mathbf{f}$, and $G^{T} G \mathbf{f}$ are, respectively, given by

$$
\begin{aligned}
(A \mathbf{f})_{i} & =5 u_{i_{1}}-u_{i_{2}}-u_{i_{3}}-u_{i_{4}}-u_{i_{5}}-u_{i_{6}}, \\
\left(B^{T} B \mathbf{f}\right)_{i} & =u_{i_{1}}-u_{i_{6}}+v_{j_{2}}-v_{j_{1}}+w_{k_{1}}-w_{k_{2}}, \\
\left(G^{T} G \mathbf{f}\right)_{i} & =\left(4 u_{i_{1}}-u_{i_{2}}-u_{i_{3}}-u_{i_{4}}-u_{i_{5}}\right)+\left(v_{j_{1}}-v_{j_{2}}\right)+\left(w_{k_{2}}-w_{k_{1}}\right) .
\end{aligned}
$$




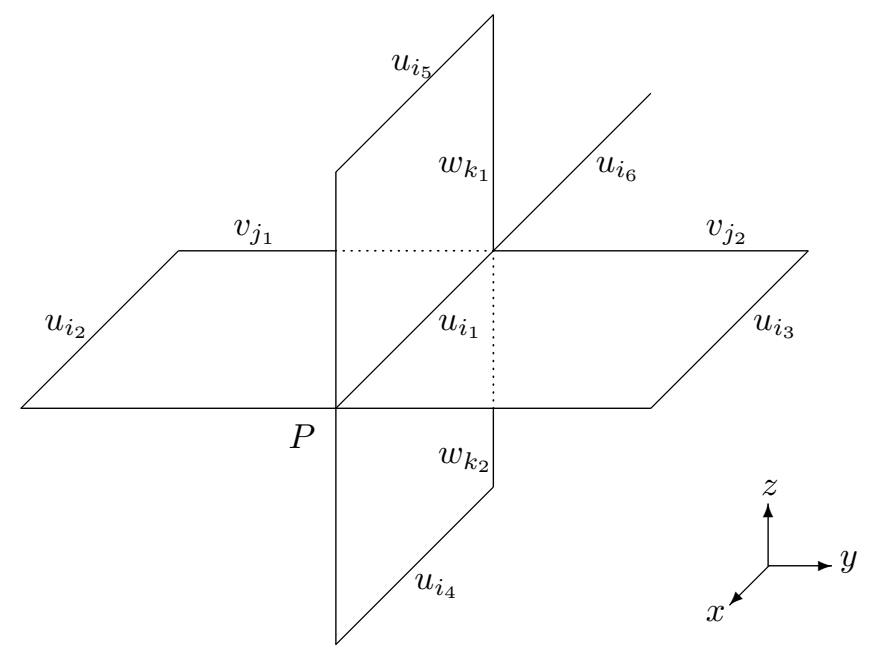
edges.

FIG. 3.2. An interior primal edge having a single-point intersection with $\partial \Omega$ and its adjacent

It is easy to check that

$$
\left(G^{T} G \mathbf{f}\right)_{i}=-\left(B^{T} B \mathbf{f}\right)_{i}+(A \mathbf{f})_{i} .
$$

We complete the proof of Theorem 3.1 by noting that any interior primal edge has either an empty intersection or a single-point intersection with $\partial \Omega$.

Before studying the eigenvalues of $G^{T} G$, we will first work on the eigenvalues of $A$. For convenience, we will let $h_{1}=1 / N_{1}, h_{2}=1 / N_{2}$, and $h_{3}=1 / N_{3}$. Note that the original triangulation is nonuniform, so $h_{1}, h_{2}$, and $h_{3}$ are not the actual nonequidistant mesh sizes along the $x$-, $y$-, and $z$-axis. However, the definitions of the matrices $G, B$, and $A$ are independent of the mesh sizes, so we can always assume that the meshes are uniform along each axis and $h_{1}, h_{2}$, and $h_{3}$ are the mesh sizes along the $x$-, $y$-, and $z$-axis, respectively. Let $k, m$, and $l$ be three integers such that $1 \leq k \leq N_{1}-1,1 \leq m \leq N_{2}-1$, and $1 \leq l \leq N_{3}-1$. Then for any fixed $k, m$, and $l$, we define

$$
\begin{aligned}
\lambda_{m l}^{1} & =4 \sin ^{2}\left(\frac{m \pi h_{2}}{2}\right)+4 \sin ^{2}\left(\frac{l \pi h_{3}}{2}\right) \\
\lambda_{k l}^{2} & =4 \sin ^{2}\left(\frac{k \pi h_{1}}{2}\right)+4 \sin ^{2}\left(\frac{l \pi h_{3}}{2}\right) \\
\lambda_{k m}^{3} & =4 \sin ^{2}\left(\frac{k \pi h_{1}}{2}\right)+4 \sin ^{2}\left(\frac{m \pi h_{2}}{2}\right) \\
\beta_{k m l} & =4 \sin ^{2}\left(\frac{k \pi h_{1}}{2}\right)+4 \sin ^{2}\left(\frac{m \pi h_{2}}{2}\right)+4 \sin ^{2}\left(\frac{l \pi h_{3}}{2}\right) .
\end{aligned}
$$

For any fixed $k, m$, and $l$, we define $\mathbf{f}_{m l}^{1}=\left(\mathbf{u}_{1}^{T}, \mathbf{v}_{1}^{T}, \mathbf{w}_{1}^{T}\right)^{T} \in \mathbb{R}^{E}$ to be a vector with only components corresponding to the interior primal edges parallel to the $x$-axis, i.e., $\mathbf{v}_{1}=\mathbf{w}_{1}=\mathbf{0}$, and the components of $\mathbf{u}_{1}$ are given by

$$
\left(\mathbf{u}_{1}\right)_{j}=\sin \left(y m \pi h_{2}\right) \sin \left(z l \pi h_{3}\right),
$$

where $\left(\mathbf{u}_{1}\right)_{j}$ is the component of $\mathbf{u}_{1}$ corresponding to the primal edge $\sigma_{j}$ which is parallel to the $x$-axis, and $y h_{2}$ and $z h_{3}$ are the $y$-coordinate and $z$-coordinate of the 
primal edge $\sigma_{j}$, respectively (with $y$ and $z$ being some positive integers). Similarly, we define $\mathbf{f}_{k l}^{2}=\left(\mathbf{u}_{2}^{T}, \mathbf{v}_{2}^{T}, \mathbf{w}_{2}^{T}\right)^{T} \in \mathbb{R}^{E}$ and $\mathbf{f}_{k m}^{3}=\left(\mathbf{u}_{3}^{T}, \mathbf{v}_{3}^{T}, \mathbf{w}_{3}^{T}\right)^{T} \in \mathbb{R}^{E}$ to be two vectors with only the components corresponding to the interior primal edges parallel to the $y$-axis and $z$-axis, respectively. Clearly, $\mathbf{f}_{m l}^{1}, \mathbf{f}_{k l}^{2}$, and $\mathbf{f}_{k m}^{3}$ are linearly independent for any fixed $k, m$, and $l$.

Furthermore, for fixed $k, m$, and $l$, we define the vector $\mathbf{g}_{k m l}^{1}=\left(\tilde{\mathbf{u}}_{1}^{T}, \tilde{\mathbf{v}}_{1}^{T}, \tilde{\mathbf{w}}_{1}^{T}\right)^{T} \in$ $\mathbb{R}^{E}(i=1,2,3)$ to be the same as $\mathbf{f}_{m l}^{1}$, but replace $\left(\mathbf{u}_{1}\right)_{j}$ in (3.3) by

$$
\left(\tilde{\mathbf{u}}_{1}\right)_{j}=\cos \left(\left(x+\frac{1}{2}\right) k \pi h_{1}\right) \sin \left(y m \pi h_{2}\right) \sin \left(z l \pi h_{3}\right),
$$

where $\left(x+\frac{1}{2}\right) h_{1}$ is the $x$-coordinate of the midpoint of the edge $\sigma_{j} \cdot \mathbf{g}_{k m l}^{2}$ and $\mathbf{g}_{k m l}^{3}$ are defined similarly. Clearly, $\mathbf{g}_{k m l}^{i}, i=1,2,3$, are linearly independent for any fixed $k, m$, and $l$.

The following theorem gives the complete spectrum and eigenvectors of $A$.

ThEOREM 3.2. For $k, m$, and $l$ satisfying $1 \leq k \leq N_{1}-1,1 \leq m \leq N_{2}-1$, $1 \leq l \leq N_{3}-1$, we have

$$
A \mathbf{f}_{m l}^{1}=\lambda_{m l}^{1} \mathbf{f}_{m l}^{1}, \quad A \mathbf{f}_{k l}^{2}=\lambda_{k l}^{2} \mathbf{f}_{k l}^{2}, \quad A \mathbf{f}_{k m}^{3}=\lambda_{k m}^{3} \mathbf{f}_{k m}^{3} ; \quad A \mathbf{g}_{k m l}^{i}=\beta_{k m l} \mathbf{g}_{k m l}^{i}, \quad i=1,2,3 .
$$

Proof. We start with the proof of the first relation in (3.5). We first consider an interior primal edge $\sigma_{j}$ having no intersection with $\partial \Omega$. If $\sigma_{j}$ is parallel to the $x$-axis and has $y$-coordinate $y h_{2}$ and $z$-coordinate $z h_{3}$, then by the definition of $A$, we have

$$
\begin{aligned}
& \left(A \mathbf{f}_{m l}^{1}\right)_{j}=\left\{6 \sin \left(y m \pi h_{2}\right)-\sin \left((y-1) m \pi h_{2}\right)-\sin \left((y+1) m \pi h_{2}\right)\right\} \sin \left(z l \pi h_{3}\right) \\
& -\sin \left(y m \pi h_{2}\right)\left\{\sin \left((z-1) l \pi h_{3}\right)-\sin \left((z+1) l \pi h_{3}\right)\right\}-2 \sin \left(y m \pi h_{2}\right) \sin \left(z l \pi h_{3}\right) .
\end{aligned}
$$

A direct computation yields

$$
\left(A \mathbf{f}_{m l}^{1}\right)_{j}=4\left\{\sin ^{2}\left(\frac{m \pi h_{2}}{2}\right)+\sin ^{2}\left(\frac{l \pi h_{3}}{2}\right)\right\} \sin \left(y m \pi h_{2}\right) \sin \left(z l \pi h_{3}\right) .
$$

This shows $\left(A \mathbf{f}_{m l}^{1}\right)_{j}=\lambda_{m l}^{1}\left(\mathbf{f}_{m l}^{1}\right)_{j}$. Now, if $\sigma_{j}$ is an interior primal edge having empty intersection with $\partial \Omega$ and is parallel to the $y$ - or $z$-axis, then $\left(\mathbf{f}_{m l}^{1}\right)_{j}=\left(\mathbf{v}_{1}\right)_{j}=0$ or $\left(\mathbf{f}_{m l}^{1}\right)_{j}=\left(\mathbf{w}_{1}\right)_{j}=0$ by definition. This implies $\left(A \mathbf{f}_{m l}^{1}\right)_{j}=0=\lambda_{m l}^{1}\left(\mathbf{f}_{m l}^{1}\right)_{j}$.

Next we consider an interior primal edge $\sigma_{j}$ having a single-point intersection with $\partial \Omega$. If $\sigma_{j}$ is parallel to the $x$-axis and has $y$-coordinate $y h_{2}$ and $z$-coordinate $z h_{3}$, then by the definition of $A$, we have

$$
\begin{aligned}
& \left(A \mathbf{f}_{m l}^{1}\right)_{j}=\left\{5 \sin \left(y m \pi h_{2}\right)-\sin \left((y-1) m \pi h_{2}\right)-\sin \left((y+1) m \pi h_{2}\right)\right\} \sin \left(z l \pi h_{3}\right) \\
& -\sin \left(y m \pi h_{2}\right)\left\{\sin \left((z-1) l \pi h_{3}\right)-\sin \left((z+1) l \pi h_{3}\right)\right\}-\sin \left(y m \pi h_{2}\right) \sin \left(z l \pi h_{3}\right) .
\end{aligned}
$$

A direct computation yields

$$
\left(A \mathbf{f}_{m l}^{1}\right)_{j}=4\left\{\sin ^{2}\left(\frac{m \pi h_{2}}{2}\right)+\sin ^{2}\left(\frac{l \pi h_{3}}{2}\right)\right\} \sin \left(y m \pi h_{2}\right) \sin \left(z l \pi h_{3}\right) .
$$

Therefore we have $\left(A \mathbf{f}_{m l}^{1}\right)_{j}=\lambda_{m l}^{1}\left(\mathbf{f}_{m l}^{1}\right)_{j}$. The same argument can be applied to prove the second and third relations in (3.5) for the case that $\sigma_{j}$ is an interior primal edge 
having an empty or a nonempty intersection with $\partial \Omega$ and is parallel to either the $y$ or $z$-axis.

We now prove the fourth relation in (3.5). First, consider an interior primal edge $\sigma_{j}$ having empty intersection with $\partial \Omega$. If $\sigma_{j}$ is parallel to the $x$-axis and has $y$-coordinate $y h_{2}$ and $z$-coordinate $z h_{3}$, with the $x$-coordinate of the midpoint of $\sigma_{j}$ being $\left(x+\frac{1}{2}\right) h_{1}$ for some integer $x$, then by the definition of $A$, we have

$$
\begin{aligned}
\left(A \mathbf{g}_{k m l}^{1}\right)_{j} & =\cos \left(\left(x+\frac{1}{2}\right) k \pi h_{1}\right)\left\{6 \sin \left(y m \pi h_{2}\right) \sin \left(z l \pi h_{3}\right)\right. \\
& -\sin \left((y-1) m \pi h_{2}\right) \sin \left(z l \pi h_{3}\right)-\sin \left((y+1) m \pi h_{2}\right) \sin \left(z l \pi h_{3}\right) \\
& \left.-\sin \left(y m \pi h_{2}\right) \sin \left((z-1) l \pi h_{3}\right)-\sin \left(y m \pi h_{2}\right) \sin \left((z+1) l \pi h_{3}\right)\right\} \\
& -\left\{\cos \left(\left(x-\frac{1}{2}\right) k \pi h_{1}\right)+\cos \left(\left(x+\frac{3}{2}\right) k \pi h_{1}\right)\right\} \sin \left(y m \pi h_{2}\right) \sin \left(z l \pi h_{3}\right),
\end{aligned}
$$

which, by a direct computation, can be written as

$$
\left(A \mathbf{g}_{k m l}^{1}\right)_{j}=\beta_{k m l} \cos \left(\left(x+\frac{1}{2}\right) k \pi h_{1}\right) \sin \left(y m \pi h_{2}\right) \sin \left(z l \pi h_{3}\right)=\beta_{k m l}\left(\mathbf{g}_{k m l}^{1}\right)_{j} .
$$

For an interior primal edge $\sigma_{j}$ having empty intersection with $\partial \Omega$ and being parallel to the $y$ - or $z$-axis, we know $\left(\mathbf{g}_{k m l}^{1}\right)_{j}=\left(\tilde{\mathbf{v}}_{1}\right)_{j}=0$ or $\left(\mathbf{g}_{k m l}^{1}\right)_{j}=\left(\tilde{\mathbf{w}}_{1}\right)_{j}=0$ by definition. Therefore

$$
\left(A \mathbf{g}_{k m l}^{1}\right)_{j}=0=\beta_{k m l}\left(\mathbf{g}_{k m l}^{1}\right)_{j} .
$$

Now, for an interior primal edge $\sigma_{j}$ having a single-point intersection with $\partial \Omega$, assume $\sigma_{j}$ is parallel to the $x$-axis and has $y$-coordinate $y h_{2}$ and $z$-coordinate $z h_{3}$, and the $x$-coordinate of the midpoint of $\sigma_{j}$ is $\left(x+\frac{1}{2}\right) h_{1}$ for $x=0$ or $x=N_{1}-1$. Then, by the definition of $A$, we have

$$
\begin{aligned}
\left(A \mathbf{g}_{k m l}^{1}\right)_{j} & =\cos \left(\left(x+\frac{1}{2}\right) k \pi h_{1}\right)\left\{5 \sin \left(y m \pi h_{2}\right) \sin \left(z l \pi h_{3}\right)\right. \\
& -\sin \left((y-1) m \pi h_{2}\right) \sin \left(z l \pi h_{3}\right)-\sin \left((y+1) m \pi h_{2}\right) \sin \left(z l \pi h_{3}\right) \\
& \left.-\sin \left(y m \pi h_{2}\right) \sin \left((z-1) l \pi h_{3}\right)-\sin \left(y m \pi h_{2}\right) \sin \left((z+1) l \pi h_{3}\right)\right\} \\
& -\cos \left(\left(x+\frac{1}{2} \pm 1\right) k \pi h_{1}\right) \sin \left(y m \pi h_{2}\right) \sin \left(z l \pi h_{3}\right),
\end{aligned}
$$

where \pm 1 is taken for $x=0$ and $x=N_{1}-1$, respectively. Using the fact that $\cos \left(\left(x+\frac{1}{2}\right) k \pi h_{1}\right)=\cos \left(\left(x-\frac{1}{2}\right) k \pi h_{1}\right)$ for $x=0$ and $\cos \left(\left(x+\frac{1}{2}\right) k \pi h_{1}\right)=\cos \left(\left(x+\frac{3}{2}\right) k \pi h_{1}\right)$ for $x=N_{1}-1$, the above relation can be written as

$$
\begin{aligned}
\left(A \mathbf{g}_{k m l}^{1}\right)_{j} & =\cos \left(\left(x+\frac{1}{2}\right) k \pi h_{1}\right)\left\{6 \sin \left(y m \pi h_{2}\right) \sin \left(z l \pi h_{3}\right)\right. \\
& -\sin \left((y-1) m \pi h_{2}\right) \sin \left(z l \pi h_{3}\right)-\sin \left((y+1) m \pi h_{2}\right) \sin \left(z l \pi h_{3}\right) \\
& \left.-\sin \left(y m \pi h_{2}\right) \sin \left((z-1) l \pi h_{3}\right)-\sin \left(y m \pi h_{2}\right) \sin \left((z+1) l \pi h_{3}\right)\right\} \\
& -\cos \left(\left(x-\frac{1}{2}\right) k \pi h_{1}\right) \sin \left(y m \pi h_{2}\right) \sin \left(z l \pi h_{3}\right) \\
& -\cos \left(\left(x+\frac{3}{2}\right) k \pi h_{1}\right) \sin \left(y m \pi h_{2}\right) \sin \left(z l \pi h_{3}\right) .
\end{aligned}
$$


This immediately leads to

$$
\left(A \mathbf{g}_{k m l}^{1}\right)_{j}=\beta_{k m l} \cos \left(\left(x+\frac{1}{2}\right) k \pi h_{1}\right) \sin \left(y m \pi h_{2}\right) \sin \left(z l \pi h_{3}\right)=\beta_{k m l}\left(\mathbf{g}_{k m l}^{1}\right)_{j}
$$

The same argument can be applied to prove (3.6) for the components of $\mathbf{g}_{k m l}^{1}$ corresponding to the primal edges parallel to the $y$ - or $z$-axis and to prove the last relation in (3.5) with $i=2,3$.

The following theorem gives the complete spectrum and eigenvectors of the discrete curl-curl operator $G^{T} G$.

TheOREM 3.3. For each triplet of integers $\{k, m, l\}$ satisfying $1 \leq k \leq N_{1}-1$, $1 \leq m \leq N_{2}-1,1 \leq l \leq N_{3}-1$, we have

$$
G^{T} G \mathbf{f}_{m l}^{1}=\lambda_{m l}^{1} \mathbf{f}_{m l}^{1}, \quad G^{T} G \mathbf{f}_{k l}^{2}=\lambda_{k l}^{2} \mathbf{f}_{k l}^{2}, \quad G^{T} G \mathbf{f}_{k m}^{3}=\lambda_{k m}^{3} \mathbf{f}_{k m}^{3} .
$$

Moreover, there exist two linearly independent vectors $\mathbf{p}_{k m l}^{1}$ and $\mathbf{p}_{k m l}^{2}$ in $\mathbb{R}^{E}$ such that

$$
G^{T} G \mathbf{p}_{k m l}^{i}=\beta_{k m l} \mathbf{p}_{k m l}^{i}, \quad i=1,2 .
$$

Proof. It is important to notice by the definitions of the matrix $B$ and the vector $\mathbf{f}_{m l}^{1}$ that, for each dual element $\tau_{j}^{\prime},\left(B \mathbf{f}_{m l}^{1}\right)_{j}=0$. This with (3.2) and (3.5) implies

$$
G^{T} G \mathbf{f}_{m l}^{1}=-B^{T} B \mathbf{f}_{m l}^{1}+A \mathbf{f}_{m l}^{1}=\lambda_{m l}^{1} \mathbf{f}_{m l}^{1} .
$$

A similar argument can be applied to show the last two relations in (3.7).

We now prove (3.8). For any fixed integers $k, m$, and $l$, we define

$$
V_{k m l}:=\operatorname{span}\left\{\mathbf{g}_{k m l}^{1}, \mathbf{g}_{k m l}^{2}, \mathbf{g}_{k m l}^{3}\right\} .
$$

Consider any $\mathbf{g}=\alpha_{1} \mathbf{g}_{k m l}^{1}+\alpha_{2} \mathbf{g}_{k m l}^{2}+\alpha_{3} \mathbf{g}_{k m l}^{3} \in V_{k m l}, \alpha_{i} \in \mathbb{R}, i=1,2,3$. We are going to find all $\alpha_{i}(i=1,2,3)$ such that $B \mathbf{g}=\mathbf{0}$. For any dual element $\tau_{j}^{\prime}$, assume its two primal edges parallel to the $x$-axis and having nonempty intersection with $\partial \tau_{j}^{\prime}$ have $x$-coordinate $\left(x-\frac{1}{2}\right) h_{1}$ and $\left(x+\frac{1}{2}\right) h_{1}$, respectively. Clearly, they have the same $y$ and $z$-coordinates, namely, $y h_{2}$ and $z h_{3}$, respectively, for some suitable integers $x, y$, and $z$. Then, by a direct computation,

$$
\begin{aligned}
\left(B \mathbf{g}_{k m l}^{1}\right)_{j} & =\left\{\cos \left(\left(x+\frac{1}{2}\right) k \pi h_{1}\right)-\cos \left(\left(x-\frac{1}{2}\right) k \pi h_{1}\right)\right\} \sin \left(y m \pi h_{2}\right) \sin \left(z l \pi h_{3}\right) \\
& =-2 \sin \left(\frac{k \pi h_{1}}{2}\right) \sin \left(x k \pi h_{1}\right) \sin \left(y m \pi h_{2}\right) \sin \left(z l \pi h_{3}\right) .
\end{aligned}
$$

Applying the same argument, we have

$$
\begin{aligned}
\left(B \mathbf{g}_{k m l}^{2}\right)_{j} & =\sin \left(x k \pi h_{1}\right)\left\{\cos \left(\left(y+\frac{1}{2}\right) m \pi h_{2}\right)-\cos \left(\left(y-\frac{1}{2}\right) m \pi h_{2}\right)\right\} \sin \left(z l \pi h_{3}\right) \\
& =-2 \sin \left(\frac{m \pi h_{2}}{2}\right) \sin \left(x k \pi h_{1}\right) \sin \left(y m \pi h_{2}\right) \sin \left(z l \pi h_{3}\right) \\
\left(B \mathbf{g}_{k m l}^{3}\right)_{j} & =\sin \left(x k \pi h_{1}\right) \sin \left(y m \pi h_{2}\right)\left\{\cos \left(\left(z+\frac{1}{2}\right) l \pi h_{3}\right)-\cos \left(\left(z-\frac{1}{2}\right) l \pi h_{3}\right)\right\} \\
& =-2 \sin \left(\frac{l \pi h_{3}}{2}\right) \sin \left(x k \pi h_{1}\right) \sin \left(y m \pi h_{2}\right) \sin \left(z l \pi h_{3}\right)
\end{aligned}
$$


Hence, $(B \mathbf{g})_{j}=0$ if and only if

$$
\alpha_{1} \sin \left(\frac{k \pi h_{1}}{2}\right)+\alpha_{2} \sin \left(\frac{m \pi h_{2}}{2}\right)+\alpha_{3} \sin \left(\frac{l \pi h_{3}}{2}\right)=0
$$

Notice that (3.9) is a condition that is independent of the choice of the dual element $\tau_{j}^{\prime}$. Hence, for any $\alpha_{i} \in \mathbb{R}(i=1,2,3)$ satisfying (3.9), we have $B \mathbf{g}=\mathbf{0}$. Let $\alpha_{1}=s$ and $\alpha_{2}=t$ for $s, t \in \mathbb{R}$. We then obtain from (3.9) that

$$
\alpha_{3}=-\frac{s \sin \left(\frac{k \pi}{2 N_{1}}\right)+t \sin \left(\frac{m \pi}{2 N_{2}}\right)}{\sin \left(\frac{l \pi}{2 N_{3}}\right)} .
$$

Then, we can express $\mathbf{g}$ as

$$
\mathbf{g}=s\left(\mathbf{g}_{k m l}^{1}-\frac{\sin \left(\frac{k \pi}{2 N_{1}}\right)}{\sin \left(\frac{l \pi}{2 N_{3}}\right)} \mathbf{g}_{k m l}^{3}\right)+t\left(\mathbf{g}_{k m l}^{2}-\frac{\sin \left(\frac{m \pi}{2 N_{2}}\right)}{\sin \left(\frac{l \pi}{2 N_{3}}\right)} \mathbf{g}_{k m l}^{3}\right) .
$$

Define

$$
\mathbf{p}_{k m l}^{1}:=\mathbf{g}_{k m l}^{1}-\frac{\sin \left(\frac{k \pi}{2 N_{1}}\right)}{\sin \left(\frac{l \pi}{2 N_{3}}\right)} \mathbf{g}_{k m l}^{3} \quad \text { and } \quad \mathbf{p}_{k m l}^{2}:=\mathbf{g}_{k m l}^{2}-\frac{\sin \left(\frac{m \pi}{2 N_{2}}\right)}{\sin \left(\frac{l \pi}{2 N_{3}}\right)} \mathbf{g}_{k m l}^{3} .
$$

Clearly, we have $B \mathbf{p}_{k m l}^{1}=B \mathbf{p}_{k m l}^{2}=\mathbf{0}$. Thus by (3.2) and (3.5), we have

$$
G^{T} G \mathbf{p}_{k m l}^{i}=-B^{T} B \mathbf{p}_{k m l}^{i}+A \mathbf{p}_{k m l}^{i}=\beta_{k m l} \mathbf{p}_{k m l}^{i}, \quad i=1,2 .
$$

We remark that Theorem 3.3 gives all the positive eigenvalues of $G^{T} G$ since the vectors $\mathbf{f}_{m l}^{1}, \mathbf{f}_{k l}^{2}, \mathbf{f}_{k m}^{3}$, and $\mathbf{p}_{k m l}^{j}$ form a complete basis for $\mathbb{R}^{E-T}$. Notice that the smallest positive eigenvalue of $G^{T} G$ is $8 \sin ^{2}\left(\frac{\pi h}{2}\right)$ which varies as $O\left(h^{2}\right)$ for sufficiently large $N$. This conclusion is important in the convergence analysis of the finite volume method proposed in [3] for Maxwell's equations with discontinuous physical coefficients.

Recall that $B$ is a discrete divergence matrix, and so $B^{T}$ represents a discrete gradient matrix. Hence, the matrix $B B^{T}$ is some sort of scalar discrete Laplacian matrix by the fact that $\nabla \cdot \nabla v=\Delta v$ for any real-valued function $v$. We have the following.

THEOREM 3.4. For any fixed integers $k, m$, and $l$ satisfying $1 \leq k \leq N_{1}-1$, $1 \leq m \leq N_{2}-1,1 \leq l \leq N_{3}-1$, there exists a vector $\mathbf{q}_{k m l} \in \mathbb{R}^{T}$ such that

$$
B B^{T} \mathbf{q}_{k m l}=\beta_{k m l} \mathbf{q}_{k m l} .
$$

Proof. For any dual element $\tau_{i}^{\prime}$, we define

$$
\left(\mathbf{q}_{k m l}\right)_{i}:=\sin \left(x k \pi h_{1}\right) \sin \left(y m \pi h_{2}\right) \sin \left(z l \pi h_{3}\right),
$$

where $x h_{1}, y h_{2}$, and $z h_{3}$ are the $x-, y$-, and $z$-coordinates of the corresponding interior primal node. Now, by a direct computation, we have

$$
\begin{aligned}
& \left(B B^{T} \mathbf{q}_{k m l}\right)_{i} \\
& \quad=6 \sin \left(x k \pi h_{1}\right) \sin \left(y m \pi h_{2}\right) \sin \left(z l \pi h_{3}\right) \\
& -\sin \left((x-1) k \pi h_{1}\right) \sin \left(y m \pi h_{2}\right) \sin \left(z l \pi h_{3}\right)-\sin \left((x+1) k \pi h_{1}\right) \sin \left(y m \pi h_{2}\right) \sin \left(z l \pi h_{3}\right) \\
& -\sin \left(x k \pi h_{1}\right) \sin \left((y-1) m \pi h_{2}\right) \sin \left(z l \pi h_{3}\right)-\sin \left(x k \pi h_{1}\right) \sin \left((y+1) m \pi h_{2}\right) \sin \left(z l \pi h_{3}\right) \\
& -\sin \left(x k \pi h_{1}\right) \sin \left(y m \pi h_{2}\right) \sin \left((z-1) l \pi h_{3}\right)-\sin \left(x k \pi h_{1}\right) \sin \left(y m \pi h_{2}\right) \sin \left((z+1) l \pi h_{3}\right) \\
& =\beta_{k m l}\left(\mathbf{q}_{k m l}\right)_{i} .
\end{aligned}
$$


We remark here that the vectors $\left\{\mathbf{q}_{\mathrm{kml}}\right\}$ in Theorem 3.4 are linearly independent, so they form the complete eigensystem of the matrix $B B^{T}$.

4. Some applications. In this section, we describe some roles of the discrete div and curl operators and some important results directly derived using the results on eigenvalues and eigenvectors of section 3. Detailed proofs of the results below are given in [4]. We remark that the constant $K$, or $K$ with subscripts, below is the generic constant independent of mesh sizes, etc.

Let $(\cdot, \cdot)$ be the standard Euclidean inner product with norm $\|\cdot\|_{2}$. We first recall two mesh and physical parameter dependent inner products introduced in [3], [6],

$$
(\mathbf{u}, \mathbf{v})_{W}:=\left(S \mathbf{u}, D^{\prime} \mathbf{v}\right) \quad \forall \mathbf{u}, \mathbf{v} \in \mathbb{R}^{F} ; \quad(\mathbf{u}, \mathbf{v})_{W^{\prime}}:=\left(S^{\prime} \mathbf{u}, D \mathbf{v}\right) \quad \forall \mathbf{u}, \mathbf{v} \in \mathbb{R}^{E},
$$

where $S=\operatorname{diag}\left(s_{i}\right), D^{\prime}=\operatorname{diag}\left(h_{j}^{\prime}\right), S^{\prime}=\operatorname{diag}\left(s_{i}^{\prime}\right)$, and $D=\operatorname{diag}\left(h_{j}\right)$ are all diagonal matrices. $s_{i}$ and $h_{j}$ are, respectively, the area of the face $\kappa_{i}$ and the length of the edge $\sigma_{j}$, and similar definitions hold for $s_{i}^{\prime}$ and $h_{j}^{\prime}$. Then we introduce two discrete circulation matrices $\mathcal{C}$ and $\mathcal{C}^{\prime}$. Following formula (2.1), we define for each interior primal and dual face $\kappa_{i}$ and $\kappa_{i}^{\prime}$,

$$
(\mathcal{C} \mathbf{u})_{\kappa_{i}}:=\sum_{\sigma_{j} \in \partial \kappa_{i}} u_{j} \tilde{h}_{j}, \quad\left(\mathcal{C}^{\prime} \mathbf{u}\right)_{\kappa_{i}^{\prime}}:=\sum_{\sigma_{j}^{\prime} \in \partial \kappa_{i}^{\prime}} u_{j} \tilde{h}_{j}^{\prime}
$$

where $\tilde{h}_{j}$ is the signed length of $h_{j}[3],[6]$; similar meanings hold for $\tilde{h}_{j}^{\prime}$ and for $\tilde{s}_{j}$ and $\tilde{s}_{j}^{\prime}$ below.

Further, we introduce two discrete flux matrices $\mathcal{D}$ and $\mathcal{D}^{\prime}$. Following the divergence theorem (2.2), we define, for each primal and dual element $\tau_{i}$ and $\tau_{i}^{\prime}$,

$$
(\mathcal{D} \mathbf{u})_{i}:=\sum_{\kappa_{j} \in \partial \tau_{i}} u_{j} \tilde{s}_{j}, \quad\left(\mathcal{D}^{\prime} \mathbf{u}\right)_{i}:=\sum_{\kappa_{j}^{\prime} \in \partial \tau_{i}^{\prime}} u_{j} \tilde{s}_{j}^{\prime} .
$$

These discrete matrices have the useful relations (cf. [3], [6], [9])

$$
\mathcal{C}=G D, \quad \mathcal{C}^{\prime}=G^{T} D^{\prime}, \quad \mathcal{D}^{\prime}=B S^{\prime} .
$$

The relations indicate that it is the matrices $\mathcal{D}^{\prime}$ and $\mathcal{C}^{\prime}$, not the matrices $B$ and $G^{T}$, that directly simulate the divergence and curl operators in the general nonuniform grids.

Discrete Sobolev inequalities. Consider two Sobolev spaces

$$
\begin{aligned}
& H_{0}(\operatorname{curl}, \operatorname{div} 0 ; \Omega)=\{\mathbf{u} \in H(\operatorname{curl} ; \Omega) ; \nabla \cdot \mathbf{u}=0 \text { in } \Omega, \mathbf{u} \times \mathbf{n}=0 \text { on } \partial \Omega\}, \\
& H_{0}(\operatorname{curl} 0, \operatorname{div} ; \Omega)=\{\mathbf{u} \in H(\operatorname{div} ; \Omega) ; \nabla \times \mathbf{u}=0 \text { in } \Omega, \mathbf{u} \times \mathbf{n}=0 \text { on } \partial \Omega\} .
\end{aligned}
$$

The Sobolev inequalities

$$
\begin{aligned}
& \|\mathbf{u}\|_{L^{2}(\Omega)} \leq K\|\nabla \times \mathbf{u}\|_{L^{2}(\Omega)} \quad \forall \mathbf{u} \in H_{0}(\operatorname{curl} ; \operatorname{div} 0 ; \Omega), \\
& \|\mathbf{u}\|_{L^{2}(\Omega)} \leq K\|\nabla \cdot \mathbf{u}\|_{L^{2}(\Omega)} \quad \forall \mathbf{u} \in H_{0}(\operatorname{div} ; \operatorname{curl} 0 ; \Omega)
\end{aligned}
$$

are essential to the mathematical analysis of Maxwell's equations [5], [6]. Accordingly, the discrete versions of these two inequalities are important in the convergence analysis 
of the numerical methods for Maxwell's equations. Corresponding to (4.5), we have [4]

$$
\begin{aligned}
\|\mathbf{u}\|_{W} \leq K\|\mathbf{u}\|_{\mathcal{C}^{\prime}} \quad \forall \mathbf{u} \in\left\{\mathbf{v} \in \mathbb{R}^{F} ; \mathcal{D} \mathbf{v}=0\right\} \\
\|\mathbf{u}\|_{W^{\prime}} \leq K\|\mathbf{u}\|_{\mathcal{C}} \quad \forall \mathbf{u} \in\left\{\mathbf{v} \in \mathbb{R}^{E} ; \mathcal{D}^{\prime} \mathbf{v}=0\right\},
\end{aligned}
$$

where $\|\cdot\|_{W}$ and $\|\cdot\|_{W^{\prime}}$ are the discrete $L^{2}$-norms induced from two inner products in (4.2), while $\|\cdot\|_{\mathcal{C}^{\prime}}$ and $\|\cdot\|_{\mathcal{C}}$ are two different discrete $H(\mathbf{c u r l} ; \Omega)$-norms, one based on the dual circulation matrix $\mathcal{C}^{\prime}$ and the other based on the primal circulation matrix $\mathcal{C}$,

$$
\|\mathbf{u}\|_{\mathcal{C}^{\prime}}^{2}=\left(S^{\prime-1} \mathcal{C}^{\prime} \mathbf{u}, D \mathcal{C}^{\prime} \mathbf{u}\right), \quad\|\mathbf{u}\|_{\mathcal{C}}^{2}=\left(S^{-1} \mathcal{C} \mathbf{u}, D^{\prime} \mathcal{C} \mathbf{u}\right) .
$$

Similarly, we can establish the discrete versions of (4.6),

$$
\begin{aligned}
&\|\mathbf{u}\|_{W^{\prime}} \leq K\|\mathbf{u}\|_{\mathcal{D}^{\prime}} \quad \forall \mathbf{u} \in\left\{\mathbf{v} \in \mathbb{R}^{E} ; \mathcal{C} \mathbf{v}=0\right\} \\
&\|\mathbf{u}\|_{W} \leq K\|\mathbf{u}\|_{\mathcal{D}} \quad \forall \mathbf{u} \in\left\{\mathbf{v} \in \mathbb{R}^{F} ; \mathcal{C}^{\prime} \mathbf{v}=0\right\}
\end{aligned}
$$

where $\|\cdot\|_{\mathcal{D}^{\prime}}$ and $\|\cdot\|_{\mathcal{D}}$ are two different discrete $H(\operatorname{div} ; \Omega)$-norms, one based on the dual flux matrix $\mathcal{D}^{\prime}$ and the other based on the primal flux matrix $\mathcal{D}$,

$$
\|u\|_{\mathcal{D}^{\prime}}^{2}=\left(V^{\prime-1} \mathcal{D}^{\prime} u, \mathcal{D}^{\prime} u\right), \quad\|u\|_{\mathcal{D}}^{2}=\left(V^{-1} \mathcal{D} u, \mathcal{D} u\right)
$$

where $V^{\prime}=\operatorname{diag}\left(A_{i}^{\prime}\right)$ and $V=\operatorname{diag}\left(A_{i}\right)$, with $A_{i}^{\prime}$ and $A_{i}$ being the volume of the dual element $\tau_{i}^{\prime}$ and the primal element $\tau_{i}$, respectively.

Solution of the div-curl equations. Following the discussion in [9], the finite volume discretization of the div-curl equations

$$
\operatorname{div} \mathbf{u}=f, \quad \operatorname{curl} \mathbf{u}=\mathbf{g}, \quad \mathbf{u} \times\left.\mathbf{n}\right|_{\Gamma}=0
$$

results in the system of linear algebraic equations of the form

$$
V^{\prime-1} \mathcal{D}^{\prime} \mathbf{u}=\bar{f}, \quad S^{-1} \mathcal{C} \mathbf{u}=\overline{\mathbf{g}} .
$$

System (4.11) is a nonsymmetric and indefinite rectangular system. One way to solve this equation is to solve its least-squares system

$$
\left(\mathcal{D}^{\prime T} V^{\prime-2} \mathcal{D}^{\prime}+\mathcal{C}^{T} S^{-2} \mathcal{C}\right) \mathbf{u}=\mathcal{D}^{\prime T} V^{\prime-1} \bar{f}+\mathcal{C}^{T} S^{-1} \overline{\mathbf{g}} .
$$

Let $\mathcal{A}$ be the coefficient matrix in (4.12). Then we can derive the following estimate for any $\mathbf{v} \in \mathbb{R}^{E}$ by using the results of section 3 (see [4] for details):

$$
K_{0}(\mathbf{v}, \mathbf{v}) \leq(\mathcal{A} \mathbf{v}, \mathbf{v}) \leq K_{1} h^{-2}(\mathbf{v}, \mathbf{v}) .
$$

By conducting more careful analyses in the derivation, one may derive more explicit bounds of $K_{0}$ and $K_{1}$ in terms of the physical coefficients, etc. Clearly, (4.13) gives an estimate of order $O\left(h^{-2}\right)$ of the condition number of the coefficient matrix in (4.12). Also, this inequality provides us with estimates on the smallest and largest eigenvalues of $\mathcal{A}$, which are useful in the convergence analysis of iterative solvers for (4.12).

As a final remark, we mention that there are other, different approaches for numerical solutions of div-curl and Maxwell's equations; see [1], [2], and the references therein. The approaches are based on the so-called de Rham finite element spaces, and the resulting discrete schemes also fulfill (3.1) and the relation $\nabla \cdot(\nabla \times \mathbf{u})=0$. 
Acknowledgments. The authors would like to thank the referees whose constructive suggestions and comments improved the presentation of the work greatly.

\section{REFERENCES}

[1] M. J. Bluck, S. P. Walker, ANd R. ORdovas, Time domain finite element methods for EM modelling, in Proc. Euro. Congr. Comput. Methods Appl. Sci. Engrg., ECCOMAS Computational Fluid Dynamics Conference, Swansea, UK, 2001, pp. 201-214.

[2] D. Boffi, A note on the discrete compactness property and the de Rham complex, Appl. Math. Lett., 14 (2001), pp. 33-38.

[3] E. Chung And J. Zou, A finite volume method for Maxwell's equations with discontinuous physical coefficients, Int. J. Appl. Math., 7 (2001), pp. 201-223.

[4] E. Chung And J. Zou, The Eigenvalues and Eigenspaces of Some Discrete Div- and CurlRelated Operators, Technical Report 2002-24 (264), Department of Mathematics, The Chinese University of Hong Kong, 2002.

[5] Z. Chen, Q. Du, AND J. Zou, Finite element methods with matching and nonmatching meshes for Maxwell equations with discontinuous coefficients, SIAM J. Numer. Anal., 37 (2000), pp. $1542-1570$.

[6] E. T. Chung, Q. Du, And J. Zou, Convergence analysis of a finite volume method for Maxwell's equations in nonhomogeneous media, SIAM J. Numer. Anal., 41 (2003), pp. $37-63$.

[7] R. A. Nicolaides, Direct discretization of planar div-curl problems, SIAM J. Numer. Anal., 29 (1992), pp. 32-56.

[8] R. A. Nicolaides AND D. Q. WANG, Convergence analysis of a covolume scheme for Maxwell's equations in three dimensions, Math. Comp., 67 (1998), pp. 947-963.

[9] R. A. Nicolaides And X. Wu, Covolume solutions of three-dimensional div-curl equations, SIAM J. Numer. Anal., 34 (1997), pp. 2195-2203. 